\title{
El género: una herramienta a favor de los derechos humanos de las mujeres y de las minorías con género no hegemónico ${ }^{1}$
}

Cecilia Barraza Morelle ${ }^{2}$

\section{Introducción}

$\mathrm{D}$ urante el 2016 en Colombia, en el marco del debate político que generó el acuerdo de paz entre el Gobierno nacional y las FarcEP, uno de los temas en los que se concentró la controversia alrededor de los contenidos de dicho documento fue el de la incorporación y uso de la categoría de análisis de género. Por tratarse de un acuerdo de paz se podría pensar que la temática de género no era un tema relevante ni mucho menos decisivo en la votación de un plebiscito a favor o en contra de dicho acuerdo. Sin embargo, para sorpresa de muchos no solo estuvo en el centro de la discusión, sino que se convirtió en

1 Este escrito es un producto del proyecto de investigación denominado Procesos de exigibilidad y justiciabilidad de los derechos humanos y el derecho internacional humanitario, en el contexto nacional e internacional. Adscrito al Grupo de Investigación Sociohumanístico Categoría A en Colciencias de la Facultad de Derecho de la Universidad Santo Tomás (Bogotá).

2 Magíster en Ciencias Políticas, licenciada en Historia y Geografía. Profesora de la Maestría en Defensa de los Derechos Humanos y el Derecho Internacional Humanitario ante Organismos, Tribunales y Cortes Internacionales de la Facultad de Derecho de la Universidad Santo Tomás (Bogotá). Correo electrónico: cbarraza@humanas.org.co 
uno de los temas que más se abordaron mediáticamente. Las discusiones y opiniones fueron diversas y profundizaron en temas como qué es género, a qué población se le aplica la categoría, frente a qué hechos, cuánto género tiene el acuerdo, incluso se realizaron conteos para señalar cuántas veces y con qué sentido se usaba el término.

Adicionalmente un grupo importante de quienes se oponían a la ratificación del acuerdo plantearon que a través de la llamada "ideología de género" que contenía el documento, se buscaba modificar la Constitución Política en lo que respecta a la definición de familia. Recientemente, en otros Estados de la región, en diferentes debates públicos a favor de los derechos de las mujeres se ha criticado el uso de la categoría género en las políticas públicas, donde se etiqueta también bajo el nombre de "ideología de género", con una clara connotación negativa ${ }^{3}$.

Este debate no es nuevo en el panorama mundial. Por ejemplo, durante la década del noventa del siglo pasado, en el marco de las discusiones que se dieron para la aprobación del Estatuto de Roma, uno de los temas álgidos estuvo vinculado justamente a la definición de género que debía incorporarse en dicho tratado. Por lo demás, fue el primer instrumento internacional que incluyó esta categoría 4 .

Considerando los debates que el concepto género ocasiona en la opinión pública, es evidente que pese a que el término se encuentra acuñado desde hace medio siglo en las ciencias sociales, aún no es aprehendido por el conjunto de la sociedad, y ello genera polémicas que dejan ver que los contenidos de la noción cuestionan el statu quo y su potencial transformador de las realidades sociales. Incluso más allá de su uso como categoría de análisis social, muestra que puede ser usado como un factor polarizador de importantes decisiones políticas en el ámbito nacional e internacional.

3 Un ejemplo reciente es el dado en Chile a raíz de los debates alrededor del proyecto de ley de despenalización parcial del aborto.

4 En el numeral 3 del artículo 7 del Estatuto de Roma se señala: "3. A los efectos del presente Estatuto se entenderá que el término género se refiere a los dos sexos, masculino y femenino, en el contexto de la sociedad. El término género no tendrá más acepción que la que antecede". 
Sin embargo, pese a los cuestionamientos que el concepto y su uso han generado en la opinión pública no es menos cierto que en los últimos 30 años el concepto ha sido incorporado en las políticas públicas, especialmente en las dirigidas a la igualdad para las mujeres, y en estos últimos años, en las políticas de reconocimiento de la diversidad de género. También, paulatinamente comienza a ser usado en las sentencias de tribunales nacionales e internacionales y se reconoce su aporte para propender por la vigencia de los derechos humanos, y particularmente de los de las mujeres.

Considerando la vigencia del concepto género, en el presente texto se busca revisar y analizar los contenidos de dicha categoría y cómo esta se constituye en una herramienta potente, aunque no única, a favor de los derechos humanos de las mujeres y de las minorías con un género no hegemónico en relación con el mandato social imperante, y para contribuir a transformar realidades. Se revisa, también, el vínculo del género con el enfoque diferencial de derechos, y los presupuestos que este enfoque debiera considerar para ser una herramienta eficaz para un desarrollo basado en la superación de las discriminaciones y garantía de igualdad sustantiva para el conjunto de la sociedad.

\section{El género como categoría de análisis}

Desde su aparición a finales de la primera mitad del siglo XX hasta la fecha, el concepto de género, como toda categoría de análisis social, ha sido construido y deconstruido. De hecho, existen diferentes aproximaciones e interpretaciones del concepto según las vertientes teóricas y epistemológicas que lo analizan. Incluso las evoluciones del concepto o alguno de los elementos que lo constituyen e interpretan han generado debates con respecto a su aceptación e incorporación como herramienta de análisis para la definición e implementación de normas y políticas públicas que contienen dicha categoría a favor de grupos discriminados. Al mismo tiempo, se ha vuelto un lugar común en el marco de las agendas públicas de los Estados contemporáneos señalar que la intervención social debe realizarse con enfoque o perspectiva de género, situación muchas veces declarativa, puesto que a la hora de ejecutar la intervención no 
se concreta dicho enfoque o perspectiva en ninguna de las etapas del ciclo de la planeación.

Dado que el propósito de esta reflexión se centra en el género como dispositivo a favor de los derechos de las mujeres y de las minorías con un género no hegemónico, se revisará la noción de género sin el interés de profundizar en las diferentes vertientes teóricas, sino haciendo énfasis en los elementos constituyentes de la categoría y en la evolución de estos para poder enfatizar en su vínculo con los derechos humanos.

En la definición más amplia, el género es un concepto que busca, a partir de la diferencia sexual, explicar las relaciones construidas entre hombres y mujeres - lo masculino y lo femenino- que se dan en un determinado momento histórico y que contribuyen a determinar parámetros socioculturales de comportamiento. En el origen del concepto género se encuentra la pregunta sobre si hay algo distinto al sexo biológico que explica el comportamiento social y la construcción de la identidad de los sujetos sexuados, frente a lo cual, el género responde que es a través del proceso de socialización y asignaciones culturales que recibe el sujeto sexuado que se construye lo femenino y lo masculino. Se genera así la diferencia entre el sexo — aquellos elementos biológicos que determinan el ser hombre o mujer-y el género — aquellos elementos socioculturales que definen la feminidad y la masculinidad-.

Si bien el concepto ha sido desarrollado en las diferentes ramas del conocimiento, el género, como categoría analítica a partir de la década de los setenta del siglo Xx, se convirtió en una herramienta no solo teórica para entender la realidad, sino sobre todo política. Esto porque permitió en parte — porque existen otros factores que se revisarán más adelante- explicar que la realidad que viven las mujeres y los hombres en sus relaciones públicas y privadas no están determinadas por su naturaleza - sexo-, sino que pueden transformarse, dado que es una construcción sociocultural — género-. Ese reconocimiento generó un cambio político a favor de la igualdad de derechos de las mujeres con respecto a los hombres, que ha sido usada a nivel global por el feminismo de diferentes vertientes. Por tanto, el género como categoría social de análisis es una herramienta para las ciencias humanas y sociales, pero sobre todo es un dispositivo político para la igualdad, cuestionador del statu quo de cualquier sistema social. Esto porque 
la discriminación que afecta a las mujeres, o a lo considerado socialmente femenino, al no fundamentarse en un determinismo biológico, es un acto de poder expresado en dominación, subordinación y opresión, producido por un sistema socialmente jerarquizado que opera en un momento histórico determinado.

Joan Scott conceptualizó que el género "es un elemento constitutivo de las relaciones sociales basadas en las diferencias percibidas entre los sexos y es una forma primaria de relaciones significantes de poder" (Scott, 1990, p. 56). Para la autora, el género se funda en cuatro áreas: representaciones simbólicas; conceptos normativos; construcción a través del parentesco, economía y organización política, y la construcción de la identidad subjetiva.

Sobre el género, Tarrés señala que:

Uno de los logros en esta construcción fue situar la categoría de género en el nivel simbólico-cultural y definirla desde ahí a partir de relaciones de poder cuyo cambio o reproducción está sujeto a factores vinculados con la historicidad de las instituciones y la organización social de los espacios donde se desarrolla la experiencia de los individuos. (Tarrés, 2013, p. 180)

Varios elementos requieren considerarse a la hora de aplicar el género. El primero es que existe una relación entre sexo y género que construye lo que se ha llamado el sistema sexo-género, que puede definirse como el conjunto de las normas y valores implícitos y explícitos, las prácticas reales y simbólicas y sus representaciones, y los imaginarios sociales que las sociedades construyen, interpretan y reinterpretan para el relacionamiento entre personas a partir de la diferencia biológica y cultural que se asigna al comportamiento sexuado de las personas.

Un segundo elemento tiene que ver con la distribución del poder en las relaciones sociales, dado que el sistema sexo-género ha producido una sociedad altamente jerarquizada caracterizada por relaciones de poder desiguales entre hombres y mujeres, o entre lo considerado el deber ser de lo femenino o masculino. Para algunos autores este tipo de jerarquización social ha sido llamado patriarcado; para otros, androcentrismo. Independientemente del nombre y de las teorizaciones alcanzadas, da cuenta de la asimetría en la distribución del poder 
real y simbólico, poder que ha recaído históricamente en los hombres que responden al modelo masculino socialmente normativo.

En consecuencia, un tercer elemento es el hecho de que el género es histórico y por ello para su análisis es necesario tomar en cuenta el contexto espacio-temporal en que se dan las relaciones sociales. Así, el análisis de género tendrá elementos constituyentes diferentes para cada momento histórico y para el tipo de sociedad que se analice. No se pueden yuxtaponer los elementos entre una cultura y otra al realizar el análisis de género. No obstante, es una constante histórica que en el análisis de género realizado a las sociedades actuales de Oriente y Occidente se ha evidenciado una asimetría en la distribución del poder que favorece a los hombres y a lo masculino dominante.

Un cuarto elemento es el carácter relacional del género. Esto implica que la lectura que se realice debe ser relacional entre lo masculino y lo femenino. Así, cuando se está aplicando la categoría de análisis de género, no se está analizando a las mujeres - lo femenino- por una parte, ni a los hombres - lo masculino- por otra, sino la forma en que interactúan relacionalmente en la sociedad, y las causas y consecuencias que tiene esa interacción para el conjunto de la sociedad, aunque esté afectando deliberadamente a un solo sujeto social o un solo sujeto social sea la causa de análisis.

Un quinto elemento hace referencia a la idea explícita en muchos estudios de género de que el sexo es un determinante biológico (natural), mientras que el género es cambiante en la medida que, al ser cultural, puede ser transformado, situación que estableció la relación sexo-género como una dicotomía. No obstante, en los noventa los estudios de Judith Butler fueron críticos frente a este elemento. Al respecto, Tarrés señala que Butler "propone una deconstrucción de la dicotomía sexo-género y muestra cómo el sexo está tan culturalmente construido como el género, de modo que la contraposición sexo/género y naturaleza/cultura pierden su razón de ser” (Tarrés, 2013, p. 186). Así el sistema sexo-género dejaría de ser una dicotomía y pasaría a ser un continuo, ambos formados culturalmente ${ }^{5}$.

5 Esta definición ha sido retomada por la CIDH en su informe del 2015 sobre población LGBT. 
Tarrés analiza que Butler cuestiona el sistema binario, dado que parte del reconocimiento de que el acceso a la realidad es mediado por la cultura y el lenguaje. Por tal razón los cuerpos están construidos culturalmente como femeninos y masculinos. Por ello, para Butler no sería posible precisar sexo y género, y se debe utilizar sexo o género o sexo-género como un continuo. Los estudios de Butler han sido de gran importancia para cuestionar la idea tradicional de que los géneros son solo dos, puesto que su teoría del continuo plantea que no hay razón para suponer que a un sexo le corresponde solo un género y que incluso, aunque el sexo fuera invariable, no necesariamente los géneros seguirán siendo dos (Tarrés, 2013, p. 187).

Más allá de estas diferencias en las aproximaciones teóricas conceptuales, la categoría género ha hecho posible leer en diferentes constructos sociales las desigualdades y el ejercicio del poder en las relaciones entre hombres y mujeres, y ello ha generado profundas transformaciones al cuestionar el orden establecido. Incluso incorporando el análisis de Butler sobre el continuo, si el sexo y el género son culturales, todo se puede de transformar y, por tanto, es posible superar la discriminación.

Por los elementos señalados, el género no ha estado ausente en las estrategias de desarrollo. Durante la década de los ochenta se incorporó esta categoría como parte de las políticas de desarrollo promovidas por las agencias del sistema de Naciones Unidas, especialmente como consecuencia del impulso que generaron las Conferencias de la Mujer promovidas por la Comisión de la Condición Jurídica y Social de la Mujer.

En un primer momento, el género en el desarrollo se asoció a la búsqueda de estrategias para empoderar a las mujeres y alcanzar la equidad entre los sexos. Se partía del reconocimiento de que las relaciones entre hombres y mujeres eran desiguales y de que era necesario nivelar el terreno de juego para garantizar oportunidades a las mujeres en el acceso a los recursos, autonomía y toma de decisiones. Durante esta primera etapa se hizo énfasis en el reconocimiento del triple rol que ejercían las mujeres al considerar sus aportes en el mundo de lo productivo, reproductivo y comunitario. Con este enfoque se dejaban de lado las políticas de desarrollo que se habían enfocado en considerar 
a las mujeres como el centro del problema, y para resolverlo, bastaba con incorporarlas a procesos productivos y generar programas de carácter asistencial, preferentemente dirigidos al binomio madre-hijo. En esta lógica las mujeres son funcionales para el desarrollo (De la Cruz, 1999, p. 56).

Actualmente, el género en el desarrollo ha tenido una variación, producto del acercamiento que se ha venido construyendo a partir de los noventa entre el desarrollo y los derechos humanos. De esta forma, el desarrollo hoy en día comprende la igualdad entre los géneros como una condición para alcanzar el desarrollo sostenible, y por tanto, las políticas públicas deben tomar en cuenta entre sus acciones aquellas dirigidas a garantizar a las mujeres acceso, control y poder sobre los recursos y sus relaciones.

La incorporación del género al desarrollo ha sido una herramienta útil para analizar y comprender los cambios que experimentan las sociedades, $\mathrm{y}$ ha brindado explicaciones a problemas sociales de diverso orden relacionados con el uso y control de los recursos, los roles de género y las políticas de redistribución, las posibilidades de regular el espacio doméstico y la comprensión de las dinámicas de los espacios privados como áreas de disputa de poder y de desigualdad, entre otras. Para ello, se ha promovido como principal herramienta la transversalización de género, o mainstreaming ${ }^{6}$.

La transversalización de género es una estrategia para alcanzar la igualdad entre los géneros, y desde este abordaje la igualdad es comprendida como las garantías que se generan para que los sujetos sociales

6 El Consejo Económico y Social de las Naciones Unidas define mainstreaming así: "Transversalizar la perspectiva de género es el proceso de valorar las implicaciones que tiene para los hombres y para las mujeres cualquier acción que se planifique, ya se trate de legislación, políticas o programas, en todas las áreas y en todos los niveles. Es una estrategia para conseguir que las preocupaciones y experiencias de las mujeres, al igual que las de los hombres, sean parte integrante en la elaboración, puesta en marcha, control y evaluación de las políticas y de los programas en todas las esferas políticas, económicas y sociales, de manera que las mujeres y los hombres puedan beneficiarse de ellos igualmente y no se perpetúe la desigualdad. El objetivo final de la integración es conseguir la igualdad de los géneros” (Ecosoc, 1997). 
accedan a la redistribución y el reconocimiento. Los aspectos relacionados con lo distributivo se refieren a las estructuras específicas de género que generan formas de injusticia en el acceso y uso de los recursos y servicios, y se incluyen la explotación basada en género y la marginación. Por su parte, el reconocimiento busca modificar los patrones culturales institucionalizados que no permiten que los miembros de una sociedad accedan a participar plenamente en la interacción social para que gocen de los beneficios que esta proporciona. La ausencia de reconocimiento genera discriminación, exclusión o marginación, y por tanto, negación o falta de garantía de derechos (Fraser, 1997). Es la interdependencia entre redistribución y reconocimiento, y su equilibrio, lo que hace posible la igualdad entre los géneros, y para lograrlo hay que partir del reconocimiento de las diferencias que existen entre los sujetos sociales, puesto que la intervención social deberá generar medidas temporales para alcanzar esa igualdad.

Incorporar el análisis de género en las políticas públicas -entendidas estas como los planes y programas de carácter gubernamental y como las normas y leyes que regulan un Estado- es ante todo una decisión política para evidenciar que las diferencias entre los géneros han ocasionado desigualdades en el ejercicio de los derechos, y es también una apuesta por modificar las estructuras de poder vigentes, ya que, al redistribuir y reconocer, se modifican las relaciones de poder, cuyo resultado último es la igualdad. Por ello, no es extraño, aunque sí peyorativo, que un sector que se opone a la incorporación del género como categoría de análisis en las políticas públicas se refiera a esto como "ideología de género", puesto que efectivamente es una apuesta política transformadora. El uso de la expresión ideología de género se originó inicialmente de la Iglesia católica y más tarde retomada por otras Iglesias cristianas. Su principal objeción es justamente al aspecto transformador que aporta el análisis de género, ya que se cuestiona que este se basa en el hecho de que el sistema sexo-género sea socialmente construido y no se reconozca como una creación divina, y por tanto, inmodificable (Lemaitre, 2016, p. 1).

El género es una herramienta clave para realizar análisis social que, al aplicarla, permite dar una explicación de por qué ocurre determinada 
situación social. Pero el género no es el sujeto de la discriminación. El sujeto de la discriminación es la persona individual o el sujeto colectivo que, al no recibir igualdad en el trato por su condición-situación de género, no accede a la distribución y uso de los recursos, y no goza del reconocimiento social suficiente para acceder de lleno a los beneficios de la interacción social o, dicho en otras palabras, se le vulneran sus derechos humanos por razones de género. Comúnmente, la desigualdad entre los géneros se ha aplicado a las relaciones entre hombres y mujeres. No obstante, la aplicación del análisis de género también permite analizar la desigualdad que afecta a aquellas personas con un género no hegemónico. Por tal razón, se ha aplicado el análisis de género a las discriminaciones que afectan a sujetos individuales y colectivos homosexuales, bisexuales, transgéneros, intergéneros u otra categoría que implique alguna forma de desigualdad de género.

El análisis de género permite leer la realidad social, pero en parte, ya que no es unívoco, puesto que el sujeto individual y colectivo no es definido ni construido solo por su género. En las sociedades se han producido y reproducido otras formas de jerarquización social que generan discriminación y vulneración de derechos y que es necesario considerar. El valioso aporte del género es que explica un tipo de relacionamiento social, el dado por el sistema sexo-género que, a pesar de su carácter histórico, no había sido considerado como un factor de desigualdad y discriminación, y por ende, de vulneración de derechos, que afecta a más de la mitad de la población.

Así, el género como categoría de análisis ha evolucionado y adquirido en estos años nuevos constructos que permiten considerarlo como un dispositivo para la igualdad política y social y que en un análisis multifactorial con otros elementos, como la etnia, la clase o la raza, tiene un potencial transformador de las desigualdades y la discriminación, y aporta, de esta forma, a la garantía de derechos y a la igualdad sustantiva.

\section{Los derechos de las mujeres y el género}

Solo en 1993, durante la Conferencia Mundial de las Naciones Unidas en Viena, se reconoció que los derechos de las mujeres son parte inalienable, 
integrante e indivisible de los derechos humanos universales, reconocimiento bastante tardío para el avance que en ese momento ya había alcanzado el derecho internacional de los derechos humanos en materia de garantía y protección de los derechos de las mujeres. Por ejemplo, hay que mencionar la Convención sobre la Eliminación de Todas las Formas de Discriminación contra la Mujer, en adelante CEDAW, firmada por los Estados partes de Naciones Unidas en 1979. Por tanto, más allá del reconocimiento formal de los derechos de las mujeres como parte del corpus de los derechos universales, ya se contaba con el principal instrumento universal a favor de la igualdad de las mujeres.

La CEDAW se fundamenta en el principio de no discriminación que implica terminar con cualquier barrera que anule o menoscabe la igualdad para las mujeres. La CEDAW define en su artículo 1 la discriminación contra la mujer como aquella que:

Denotará toda distinción, exclusión a restricción basada en el sexo que tenga por objeto o por resultado menoscabar o anular el reconocimiento, goce o ejercicio por la mujer, independientemente de su estado civil, sobre la base de la igualdad del hombre y la mujer, de los derechos humanos y las libertades fundamentales en las esferas política, económica, social, cultural y civil o en cualquier otra esfera. (CEDAW, 1979, p. 2)

En estricta correlación con el artículo 1, la CEDAW en su artículo 2 se refiere a la igualdad, al señalar que los Estados partes no solo condenarán la discriminación, sino que generarán políticas para garantizar la igualdad formal y de $\mathrm{facto}^{7}$. Incluso más allá, en su artículo 4, esti-

7 En el artículo 2 señala las siguientes medidas: “a) Consagrar, si aún no lo han hecho, en sus constituciones nacionales y en cualquier otra legislación apropiada el principio de la igualdad del hombre y de la mujer y asegurar por ley u otros medios apropiados la realización práctica de ese principio; b) adoptar medidas adecuadas, legislativas y de otro carácter, con las sanciones correspondientes, que prohíban toda discriminación contra la mujer; c) establecer la protección jurídica de los derechos de la mujer sobre una base de igualdad con los del hombre y garantizar, por conducto de los tribunales nacionales o competentes y de otras instituciones públicas, la protección efectiva de la mujer contra todo acto de discriminación; d) abstenerse de incurrir en todo acto 
pula que no puede considerarse discriminación a aquellas medidas de carácter temporal que los Estados partes implementen para acelerar la igualdad de facto entre el hombre y la mujer. Estas medidas temporales y sus respectivas políticas han sido interpretadas como el mainstreaming o enfoque de género en las políticas.

Dado el contexto histórico al momento de elaboración de la CEDAW, el texto de la Convención no hace referencia explícita al concepto de género. No obstante, señala en su artículo 5 que los Estados partes deberán tomar medidas para:

Modificar los patrones socioculturales de conducta de hombres y mujeres, con miras a alcanzar la eliminación de los prejuicios y las prácticas consuetudinarias y de cualquier otra índole que estén basados en la idea de la inferioridad o superioridad de cualquiera de los sexos o en funciones estereotipadas de hombres y mujeres. (CEDAW, 1979, p. 4)

Como se verá más adelante, los elementos mencionados en este artículo de la Convención constituyen categorías básicas del análisis de género.

En los últimos años el reconocimiento de los derechos humanos de las mujeres ha tenido un desarrollo progresivo tanto en el interior del sistema de Naciones Unidas como del sistema interamericano. Estos instrumentos normativos, como la jurisprudencia proferida, coinciden en referirse a la discriminación que afecta a las mujeres como una discriminación histórica, idéntico concepto usado en otros instrumentos internacionales que refieren a la discriminación histórica, que, por ejemplo, afecta a la población indígena u originaria y a la población afrodescendiente y otras diferentes formas de racismo, dado que la discriminación hacia las mujeres y el racismo han estado presentes a través

a práctica de discriminación contra la mujer y velar porque las autoridades e instituciones públicas actúen de conformidad con esta obligación; e) tomar todas las medidas apropiadas para eliminar la discriminación contra la mujer practicada por cualesquiera personas, organizaciones o empresas; f) adaptar todos las medidas adecuadas, incluso de carácter legislativo, para modificar o derogar leyes, reglamentos, usos y prácticas que constituyan discriminación contra la mujer; g) derogar todas las disposiciones penales nacionales que constituyan discriminación contra la mujer”. 
de la historia en todas las formas de organización sociopolítica y económica, sin que se haya logrado superar del todo (Garay, 2008, p. 271).

\section{El Comité de la CEDAw y el género}

Para la Convención y para las interpretaciones del Comité de la CEDAW, es claro que el sujeto de la discriminación es la mujer, en quien las características atribuibles a su sexo y a su género son las que generan la discriminación. El Comité de la CEDAw ha profundizado en la temática de género, particularmente en la Recomendación General 28 y en la reciente Recomendación General 35, y ello permitió una interpretación progresiva que ha fortalecido los estándares de derechos humanos.

En particular, la Recomendación General 28 aclara el alcance y el significado del artículo 2 de la CEDAW. En su parágrafo 5 señala:

El término sexo se refiere aquí a las diferencias biológicas entre el hombre y la mujer. El término género se refiere a las identidades, las funciones y los atributos construidos socialmente de la mujer y el hombre y al significado social y cultural que la sociedad atribuye a esas diferencias biológicas, lo que da lugar a relaciones jerárquicas entre hombres y mujeres y a la distribución de facultades y derechos en favor del hombre y en detrimento de la mujer. El lugar que la mujer y el hombre ocupan en la sociedad depende de factores políticos, económicos, culturales, sociales, religiosos, ideológicos y ambientales que la cultura, la sociedad y la comunidad pueden cambiar. (Comité CEDAW, 2010, p. 2)

Este punto entonces permite afirmar que la discriminación por motivos de género está contenida en el artículo 1 de la Convención, lo que supone que los Estados partes tienen la obligación de no discriminar por razones de género a las mujeres, ya sea por acción u omisión, y deben tomar las medidas para prevenir tal proceder, ya sea en el interior del Estado o entre particulares. Incluso, el Comité señala que la intervención que puede realizar el Estado partiendo del supuesto de que las políticas son neutras puede ser considerado discriminación por omisión, si priva a las mujeres de sus derechos o no toma en cuenta las desventajas por motivos de género (Comité CEDAW, 2010, p. 2). 
La Recomendación General 28, también avanza en desarrollar el concepto de discriminación directa e indirecta al señalar que la discriminación directa contra las mujeres

Es la que supone un trato diferente fundado explícitamente en las diferencias de sexo y género. La discriminación indirecta contra la mujer tiene lugar cuando una ley, una política, un programa o una práctica parece ser neutra por cuanto se refiere tanto a los hombres como a las mujeres, pero en la práctica tiene un efecto discriminatorio contra la mujer porque las desigualdades preexistentes no se han tenido en cuenta en la medida aparentemente neutra. (Comité CEDAW, 2010, p. 4)

Asimismo, el Comité vuelve a señalar, como ya lo había hecho en la Recomendación General 19, que la violencia contra las mujeres por motivos de género ${ }^{8}$ es una forma de discriminación y que puede constituir una violación a la Convención, aunque la CEDAW no mencione expresamente el concepto de violencia.

Es interesante para los propósitos de este escrito mencionar que esta recomendación iguala los términos principio de igualdad entre el hombre y el de la mujer con el de igualdad entre los géneros. Además, introduce el concepto de interseccionalidad, al señalar que:

La discriminación de la mujer por motivos de sexo y género está unida de manera indivisible a otros factores que afectan a la mujer, como la raza, el origen étnico, la religión o las creencias, la salud, el estatus, la edad, la clase, la casta, la orientación sexual y la identidad de género. La discriminación por motivos de sexo o género puede afectar a las mujeres de algunos grupos en diferente medida o forma que a los hombres. (Comité CEDAw, 2010, p. 5)

Por su parte, la Recomendación General 35, emitida en el 2017, proporciona a los Estados orientaciones complementarias a la Recomendación

8 La Recomendación General 19 define la violencia basada en el género como aquella dirigida contra una mujer por el hecho de ser mujer o que la afecta desproporcionadamente. 
General 19 para avanzar en la eliminación de la violencia de género contra las mujeres. Complementa la aplicación del concepto de interseccionalidad al incorporar tácitamente variables como:

Etnia/raza, situación indígena o minoritaria, color, condición socioeconómica y/o casta, idioma, religión o creencias, opiniones políticas, origen nacional, estado civil y/o maternidad, edad, situación de salud, discapacidad, propiedad, ser lesbiana, bisexual, transgénero o intersexual, analfabetismo, trata de mujeres, conflicto armado, buscar asilo, ser refugiado, desplazamiento interno, apátrida, migración, jefas de hogares, viudez, vivir con VIH, las mujeres que luchan por sus derechos, incluidos los defensores de los derechos humanos. ${ }^{9}$ (Comité CEDAw, 2017, p. 4)

El Comité reconoce que la violencia de género - expresión de discriminación - afecta a las mujeres en diferentes grados y con un efecto agravante. También señala que la discriminación está presente en todos sus ciclos de vida, por tanto, incluye a las niñas como víctimas de violencia de género.

En el parágrafo 19 de la Recomendación 35, se expresa que la violencia de género contra las mujeres se encuentra enraizada en

Factores relacionados con el género, como la ideología del derecho y el privilegio de los hombres sobre las mujeres, las normas sociales relativas a la masculinidad, la necesidad de asegurar el control o el poder masculino, prevenir, desalentar o castigar lo que se considera un comportamiento femenino inaceptable. ${ }^{10}$ (Comité CEDAW, 2017, p. 6)

Estas situaciones permiten la aceptación social y por ende la impunidad frente a estos casos.

También es de destacar que esta recomendación general enfatiza en la necesidad de garantizar una reparación efectiva a las víctimas de violencia de género. Dicha reparación implica compensación económica

9 Traducción al español propia, no oficial.

10 Traducción al español propia, no oficial. 
y acceso a servicios de salud y legales, así como recuperación de la víctima, satisfacción y garantías de no repetición, en concordancia con otras recomendaciones del mismo Comité. Para el caso colombiano en este contexto es interesante recalcar que el Comité señala que los Estados deben establecer fondos de reparación específicos o incluir asignaciones para la violencia de género contra la mujer dentro de los fondos existentes, incluso en el marco de los mecanismos de justicia de transición (Comité CEDAW, 2017, p. 7).

Como se ha podido observar, el Comité de la CEDAw, a través de sus recomendaciones generales, ha fortalecido el corpus original, para permitir con ello una interpretación progresiva que ha fortalecido los estándares de derechos humanos y su relación directa con el uso del concepto de género.

\section{El Sistema Interamericano y el género}

El Sistema Interamericano de Derechos Humanos tampoco se ha quedado atrás en incorporar el concepto de género, particularmente en el tratamiento de las violencias contra las mujeres. Se destaca el caso Raquel Martín de Mejía vs. Perú, en el cual la Comisión Interamericana de Derechos Humanos, en 1996, calificó la violación sexual como tortura. Más tarde, en 2001, con el caso María Da Penha Maia Fernándes vs. Brasil, la Comisión fortaleció sus argumentos, particularmente sobre la debida diligencia (Fernández, 2017, p. 5).

La Corte Interamericana, por su parte, en 2006, con el caso Penal Miguel Castro-Castro vs. Perú, inició su intervención en las temáticas de género. Sin embargo su hito más relevante lo constituye la Sentencia González y Otras vs. México, caso más conocido bajo el nombre de Campo Algodonero. Esta sentencia avanzó en lo que respecta a la debida diligencia de los Estados y en la sanción y medidas de reparación. Señaló explícitamente que las investigaciones debían tener perspectiva de género y contar con personal calificado en la materia. Uno de los avances más relevantes del caso Campo Algodonero se relaciona con la definición que proporciona la Corte con respecto de los estereotipos de género, al definirlos como una preconcepción de atributos o características propias que son o se espera que sean realizados por 
hombres y mujeres, y que en ellos se encuentra la causa y consecuencia de la violencia contra las mujeres. Con posterioridad, en otras sentencias la Corte ha seguido usando el concepto de estereotipos de género, incluidos los relacionados con orientación sexual, y señala que estos estereotipos pueden afectar tanto al hombre como a la mujer de manera desproporcionada (Fernández, 2017, p. 8).

\section{La normativa colombiana y el género}

Si bien la Constitución Política de Colombia en su texto original no utiliza el concepto género, este sí ha sido incorporado con la modificación del artículo 107, mediante acto legislativo dado en 2009, referente a los partidos y movimientos políticos, en el cual se señala que uno de sus principios rectores para su organización será la equidad de género. También algunas leyes, tales como la Ley 248 de 1995, Ley 823 de 2003, Ley 984 de 2005 y Ley 1098 de 2006, entre otras, utilizan la expresión género o perspectivas de género (Campillo-Vélez, 2013).

En Colombia existe un acumulado importante, particularmente de las Altas Cortes, con respecto a garantías de igualdad para las mujeres en desarrollo del artículo 13 y 43 de la Constitución Política de 1991. La Corte Constitucional se ha pronunciado sobre el tema en diversas sentencias vinculadas con derechos de familia, educación, igualdad, intimidad y dignidad, participación, salud, desarrollo de la libre personalidad, derecho a una vida libre de violencia, trabajo y derechos laborales, conflicto armado, desplazamiento, mujeres privadas de la libertad, derechos sexuales y reproductivos de las mujeres, mujeres indígenas desde una perspectiva de género, entre otras ${ }^{11}$.

En particular, la Corte Constitucional relaciona las discriminaciones en el ejercicio de los derechos de las mujeres como materias de género al perpetuar estereotipos culturales. La Corte también ha asumido las diferencias entre sexo y género, y les asigna al primero un componente biológico y al segundo, un componente social, impuesto

11 Para mayor información se puede consultar la página web: http://www.corteconstitucional.gov.co/inicio/equidaddegenero.php 
a través de roles. En muchos casos utiliza los conceptos sexo y género como sinónimos, aunque aclara que, cuando lo hace, es porque comprenden la dimensión biológica y social de manera integrada. También, esta Corte se ha pronunciado sobre las múltiples discriminaciones, pues señala que la discriminación que afecta a las mujeres basada en género se expresa junto con otras discriminaciones como la étnica y la etaria (Campillo-Vélez, 2013).

\section{La discriminación múltiple}

Como ya se planteó, tanto el Sistema Universal —a través del Comité de la CEDAW- como las instancias del Sistema Interamericano y la Corte Constitucional colombiana han reconocido que la discriminación puede llegar a ser múltiple. En efecto, la identidad del sujeto individual o colectivo es múltiple, ya que se construye a partir de un conjunto de rasgos, atributos y características que lo diferencian de otros. El género ${ }^{12}$ es un factor de discriminación para el ejercicio de los derechos, pero no es el único, puesto que en la construcción de identidad del sujeto entran a jugar otros rasgos que son determinantes para generar o no discriminación, como la clase, posición educativa, ubicación urbana/rural, pertenencia étnica, etaria, entre otros. Dichos factores pueden ser condicionantes o situacionales. En ambos casos es necesario un abordaje desde las multiplicidades de la discriminación para poder brindar una atención integral del sujeto social que apunte a garantizar la igualdad.

La discriminación múltiple se refiere a aquella que es producida por diversas causas o motivos - factores - de discriminación que actúan de manera conjunta y simultánea. Por tal razón, si se analiza la discriminación tomando en cuenta solo uno o solo una parte de los motivos de discriminación, se corre el riesgo de realizar un análisis parcializado de la realidad. La discriminación múltiple se puede clasificar en dos tipos: la acumulativa y la interseccional. En los casos en que

12 Es necesario tener presente que la orientación sexual tampoco es un asunto solo determinado por el género. 
la discriminación se debe a la existencia, al mismo tiempo, de varios motivos o causas, en la cual un motivo se agrega a otro, se clasifica como discriminación múltiple acumulativa. Por su parte, la discriminación múltiple interseccional hace referencia a los casos en los cuales diversos motivos o causas de discriminación, al actuar de manera conjunta y simultánea, producen una nueva forma de discriminación. La discriminación múltiple acumulativa puede ejemplificarse en el caso de una mujer en situación de desplazamiento que busca trabajo. En el primer lugar donde la entrevistan es rechazada porque se presume que no cuenta con las condiciones físicas para desempeñar el cargo, y en el segundo lugar, es rechazada, pues aducen problemas de seguridad por encontrarse en situación de desplazamiento. Hay dos motivos de discriminación que se añaden: el primero, por ser mujer, y el segundo, por su situación de desplazamiento. Mientras que la discriminación múltiple interseccional se podría ejemplificar en la discriminación que sufre una mujer indígena, en cuanto la discriminación que la afecta es consecuencia de la interacción de ser mujer e indígena, en la cual las vivencias de mujer e indígena coactúan, no de manera sumatoria sino reproductiva, y se genera entonces una nueva forma de discriminación (De Lama, 2013, pp. 274 y 275).

Estos factores — causas y motivos- que generan discriminación y desigualdad se fundamentan en las diferentes formas de jerarquización social que se construyen y que responden a un momento histórico determinado. Las formas de jerarquización social actúan según qué tan cerca o tan lejos se esté del paradigma sociocultural de los sujetos predominantes o dominantes en una sociedad. Es decir, cuantas más semejanzas con los sujetos sociales predominantes o dominantes, más garantías de igualdad, puesto que se hace parte de los "iguales", y viceversa: cuantas menos semejanzas, mayor desigualdad, pues se constituye la otredad, y es justamente la construida a partir de las diferencias entre hombres y mujeres una de las otredades más evidentes en las sociedades.

La asimetría en las relaciones de poder que genera la jerarquización social y que se expresa en la desigualdad entre los sujetos sociales es multifactorial y varía según el contexto; por ejemplo, las mujeres han sido históricamente discriminadas por razones de género, pero las formas de discriminación de género no son necesariamente las mismas 
en el siglo XIX que en el XXI, en la cultura islámica, en la cultura judeocristiana o en las culturas indígenas. Tampoco puede equipararse la discriminación de género que afecta a las mujeres colombianas en la segunda década del siglo XXI con las mismas desigualdades, como si fueran un todo y único conjunto social, ya que la discriminación de género estará mediada por otras formas de discriminación que afectarán a las mujeres (y a lo considerado femenino) y que interactuarán como un dispositivo acumulador de la discriminación o como dispositivo de otras nuevas discriminaciones.

\section{El enfoque diferencial de derechos}

Actualmente es recurrente en el campo de las ciencias sociales utilizar el término enfoque diferencial como herramienta para abordar los problemas sociales y las estrategias para afrontarlos; se utiliza también para estudios cuantitativos como cualitativos, y permite realizar análisis desagregados para dar cuenta de la diversidad que compone la realidad social, que particularmente incluyen variables de análisis, como sexo, género, etnia, edad, urbano-rural, entre otras.

En materia de enfoque diferencial se han registrado importantes avances en desagregación de información cuantitativa. También, se registran avances en análisis cualitativos. En el caso concreto de los estudios de género, se ha avanzado en áreas diversas, tanto de la vida pública como privada, y destacan los estudios sobre trabajo, mercado laboral, informalidad, economía del cuidado, participación social y política, educación, salud, violencia, entre otros. Incluso en algunos casos se ha avanzado en estudios que incorporan otros motivos de discriminación, que han permitido no solo caracterizar las diferencias entre hombres y mujeres generadoras de discriminación, sino también las diferencias entre las mujeres, a través de la incorporación de variables, como la etnia, la edad o el nivel educativo.

Si bien las desigualdades existentes en la sociedad generadoras de discriminación han sido abordadas por las políticas públicas desde diferentes perspectivas con el propósito de alcanzar el desarrollo, en estos últimos años se ha posicionado como una alternativa la del enfoque diferencial de derechos. Particularmente en América Latina, 
se ha recurrido a la aplicación del enfoque diferencial de derechos como mecanismo para la superación de las múltiples discriminaciones que afectan a los sujetos sociales. No sobra señalar que, tanto en la incorporación del género en particular como del enfoque diferencial de derechos en general en las políticas públicas, han desempeñado un papel fundamental los movimientos sociales y de derechos humanos, en especial el de mujeres y feministas.

El enfoque diferencial de derechos busca considerar a los sujetos desde la diversidad y diferencias que los constituyen, es decir, desde el reconocimiento de las identidades múltiples y las diversas formas de discriminación que pueden generar; parte de la obligación del Estado de proteger los derechos fundamentales, y lo aplica al conjunto de la intervención social —análisis, actuación y evaluación- que se realiza desde el Estado a través de políticas públicas. Básicamente se busca que el sujeto social de la intervención pública sea considerado un sujeto con derechos y con capacidad de exigibilidad, en el que el Estado tiene la obligación y responsabilidad de actuar. Sin duda, este enfoque es una clara evidencia del vínculo entre derechos humanos y desarrollo, en el cual la guía del desarrollo se sustenta en el marco conceptual del derecho internacional de los derechos humanos (Abramovich, 2004, p. 7).

Discursivamente en las políticas públicas que se diseñan e implementan en Colombia ${ }^{13}$ suele separarse el enfoque diferencial del enfoque de género, probablemente con la intención política de enfatizar en el enfoque de género como condición sine qua non de la intervención pública que se realice, aunque no se puede desconocer que el uso del género como categoría de análisis es un herramienta del enfoque diferencial, puesto que el reconocimiento de los derechos de las mujeres y de las minorías con género no dominante se hace efectivo a través del

13 Un claro ejemplo se encuentra en los Lineamientos de la Política Pública Nacional de Equidad de Género para las Mujeres, y en el Conpes 161, que contiene la política pública para las mujeres del Gobierno nacional para el periodo 2013-2016. También, el Acuerdo Final para la Terminación del Conflicto y la Construcción de una Paz Estable y Duradera firmado por el Gobierno de Colombia y las Farc-EP, diferencia tres enfoques para garantizar derechos: de género, territorial y diferencial. 
análisis de género en todo el ciclo de la planeación ${ }^{14}$. El género es un componente del enfoque diferencial de derechos.

El enfoque diferencial de derechos, en teoría, permite implementar acciones afirmativas que contribuirían a garantizar la igualdad sustantiva y, finalmente, a transformar los imaginarios sociales y culturales que están en la base de las diversas formas de discriminación. No obstante, llevar el enfoque a la implementación de la intervención social no es tarea fácil. Es posible plantear que a la hora de avanzar en la intervención social, el enfoque diferencial de derechos -incluido el de género- comienza a perder la riqueza del reconocimiento de las identidades múltiples que interactúan socialmente, y por ende, de las discriminaciones múltiples que lo afectan. Ello genera una intervención que fragmenta o compartimenta al sujeto social y una sectorialización de la intervención que muchas veces profundiza la discriminación, dado que no logra atender las múltiples discriminaciones de manera articulada o enfrenta a los grupos sociales por el acceso a los recursos que proporciona la intervención. Esto no significa que se quiera afirmar que el enfoque diferencial de derechos no se constituya en una vía efectiva para garantizar que las políticas públicas contribuyan a superar las desigualdades, solo que en la práctica presenta tensiones que requieren ser atendidas.

El enfoque diferencial de derechos —incluido el de género- en las políticas públicas tiene un gran potencial, puesto que parte de reconocer que los grupos discriminados, y por tanto excluidos, de los beneficios del desarrollo tienen derechos, y de entrada les otorga un poder para la exigibilidad. Además este enfoque asume como punto de partida que los derechos son interdependientes, situación que implica que el abordaje de las políticas debe ser multisectorial. Al respecto,

14 Dado que reconoce la existencia de una desigualdad de género entre hombres y mujeres y las minorías que adquieren un género no hegemónico al mandato social imperante, pero también reconoce a las mujeres y a las minorías con género no hegemónico como un conjunto heterogéneo, los cuales no son posibles de generalizar, puesto que la vivencia del género está determinada por la interacción de otras diferencias y diversidad sociocultural que pueden generar o no discriminaciones múltiples. 
Abramovich plantea que "los derechos no nos dicen mucho acerca del contenido de las políticas, pero sí pueden decirnos algunas cosas sobre su orientación general y nos brindan un marco de conceptos que debe guiar su formulación y su implementación” (Abramovich, 2004, p. 8).

En otras palabras, el enfoque diferencial de derechos no señala las acciones que deben emprenderse, sino las orientaciones que deben seguirse en las políticas públicas, orientaciones que pueden resumirse en las obligaciones de los Estados de respetar, proteger, garantizar y promover el derecho o los derechos que están siendo abordados a partir de los estándares del derecho internacional de los derechos humanos. Como lo señala Abramovich, citando a Van Hoof y a Asbjorn Eide:

Las obligaciones de respetar se definen por el deber del Estado de no injerir, obstaculizar o impedir el acceso el [sic] goce de los bienes que constituyen el objeto del derecho. Las obligaciones de proteger consisten en impedir que terceros injieran, obstaculicen o impidan el acceso a esos bienes. Las obligaciones de garantizar suponen asegurar que el titular del derecho acceda al bien cuando no puede hacerlo por sí mismo. Las obligaciones de promover se caracterizan por el deber de desarrollar condiciones para que los titulares del derecho accedan al bien. (Abramovich, 2004, p. 16)

En particular, para la concreción del principio de igualdad y no discriminación, el enfoque diferencial de derechos permite avanzar hacia la igualdad sustantiva (igualdad material), puesto que exige al Estado la protección especial de los grupos afectados por condiciones-situaciones de discriminación histórica, que deben concretarse en medidas efectivas e inmediatas y que transformen esa desigualdad, con base en los desarrollos del derecho internacional de los derechos humanos. Concurrente con esto, supone desarrollar también los mecanismos para la participación, tomando en cuenta las particularidades de los grupos excluidos y las medidas especiales para garantizar la participación, así como el acceso a la información pública y a los procesos de rendición de cuentas (Abramovich, 2004, p. 32).

El enfoque de derechos es un deber ser, un paradigma de desarrollo que busca alcanzar el desarrollo a través de la garantía de los derechos humanos; orienta el camino que las políticas públicas deben seguir; 
responde para qué alcanzar el desarrollo y por qué hacerlo, para que el sujeto social ejerza sus derechos como garantía para la superación de la desigualdad porque las personas son sujetos de derechos exigibles al Estado, y el Estado tiene la obligación de garantizárselos. No obstante, no define cómo hacerlo, aunque dice qué aspectos deben considerarse para garantizar que lo que se realice cuente con participación, información pública y rendición de cuentas. El Estado es el que define - con la participación de los sujetos sociales-qué acciones en concreto se implementarán para garantizar los derechos, y cuáles no.

Teóricamente durante el proceso de diseño de una política puede aplicarse un test de estándares de derechos humanos y determinar el cumplimiento o no de esos estándares. Sin embargo, a la hora de implementar la política no es tan fácil señalar qué acciones se van a priorizar — con la participación de los involucrados- para garantizar que los estándares de derechos humanos se alcancen. Situación que se complejiza aún más por los recursos limitados, incluyendo tiempo y debilidades en la inversión económica que se realiza.

Tampoco será fácil realizar la evaluación de la política, puesto que lo que se requiere medir no es solo lo que tangiblemente se realizó, sino aquello que transformó al sujeto social de la política como garante del derecho. Es decir, la ejecución fue capaz de permitir la superación de la vulneración y los sujetos sociales superaron su situación-condición de discriminación.

Por ello, sin duda los mayores desafíos del enfoque se encuentran en la definición de las acciones concretas que se deberán aplicar para garantizar derechos, puesto que entran a jugar en las decisiones aspectos como recursos, actores involucrados, prioridades institucionales, entre otros. Pero tal vez la mayor complejidad está dada por el vínculo entre el reconocimiento de las múltiples discriminaciones que afectan al sujeto social, la interdependencia de los derechos y el fin último de garantizar la superación de la discriminación.

Por ejemplo, en una política para la igualdad de derechos entre hombres y mujeres en Colombia, no solo deberán considerarse las diferencias entre mujeres y hombres en el acceso y uso de los recursos y el reconocimiento que gozan en la sociedad, sino que será necesario considerar las diferencias que existen entre las mujeres que componen 
la población colombiana. El objeto de la política son las mujeres colombianas en su totalidad, incluso es posible que en general la exigibilidad de derechos sea la misma: derechos a una vida libre de violencia, derecho a la salud, a la participación, etc. Es decir, se está reconociendo la diversidad del sujeto y la interdependencia de derechos, pero la acción dirigida a una niña urbana en edad escolar para garantizarle sus derechos será muy distinta de la que se destina a una mujer indígena desplazada. A ambas habrá que garantizarles sus derechos a la igualdad como mujeres - parte de un colectivo históricamente discriminado-, pero en una deberá considerarse la variable etaria y en la otra se considerarán su origen étnico y su situación de desplazamiento de manera interdependiente, reconociendo la discriminación múltiple; y aunque se consideren las diferencias a la hora de aplicar la intervención, no hay garantía de transformación de la realidad.

En los casos en que se busque garantizar más de un derecho o en los cuales los sujetos a los que se les dirige la política son diversos y con interseccionalidad de discriminaciones, la definición es aún más compleja, puesto que la intervención deberá ser múltiple sobre todo si se busca la superación de una discriminación histórica. A esto hay que agregarle el hecho de que en general en América Latina las políticas públicas no han tenido vocación de derecho, sino un fuerte componente asistencial u objetivando al sujeto, siendo la persona un objeto al servicio del desarrollo y no el centro de este.

Por tanto, no es solo necesario aplicar a las políticas públicas unos test para verificar si cumplen o no con los estándares internacionales de derechos, ni puede reducirse a que los tribunales nacionales e internacionales se pronuncien si los Estados están cumpliendo o no, o que los sujetos realicen exigibilidad de derechos a través de los tribunales. Por tal razón, para que las acciones concretas que se realicen garanticen los derechos del sujeto social focalizado y generen la superación de la desigualdad identificada, sería necesario contemplar los siguientes supuestos:

a. La sociedad en su conjunto ha realizado un pacto social para superar las desigualdades como apuesta de desarrollo, es decir, existe un compromiso social con la igualdad. Para ello, 
es necesario un diálogo social de carácter integral promovido por el Estado con la participación de los diversos grupos sociales, que permita apuestas globales de desarrollo y no fraccione o compartimente a los sujetos. Es decir, que el diálogo social genere un poder que sume positivo, en el cual las políticas que se implementen se reviertan en una ganancia exponencial para todos los sujetos discriminados y no solo para aquellos que reciben el beneficio directo. Así se reducen las competencias entre desiguales por escasos recursos y el temor de los que ya han alcanzado los beneficios del desarrollo de perder sus derechos.

b. Aplicación de test de estándares en derechos humanos. Las políticas deben garantizar los estándares mínimos de derechos humanos, y no puede justificarse una política que por considerar la diversidad cultural no respete dichos mínimos.

c. Se ha difundido al conjunto de la sociedad la información sobre la intervención, incluidos objetivos, presupuesto y prioridades, y se realizan procesos de rendición de cuentas periódicos y participativos.

d. La política pública responde a un criterio de universalidad pero focalizado, es decir, está dirigida a un grupo o colectivo al cual se le ha vulnerado un derecho o más de uno y se encuentra en situación de vulnerabilidad o discriminación. Los objetivos y los resultados son de carácter universal, pero la definición de acciones es focalizada, y para realizar dicha focalización es necesario tomar en cuenta los otros factores de discriminación que intervienen.

e. La política define con precisión qué derechos debe garantizar para superar la discriminación que busca atender, pero la intervención en su conjunto debe tomar en cuenta otras formas de discriminación que afectan al sujeto, y debe ser complementaria con otras intervenciones. 
f. Responden a realidades territoriales. Las acciones que implementen las políticas se deben definir en el territorio físico o simbólico. Esto significa que siendo el mismo objetivo la acción depende de las múltiples discriminaciones que interfieren en el sujeto y que se viven en un territorio. El territorio cumple un papel fundamental como espacio para la definición del que se hará en la intervención. En esta lógica las unidades administrativas requieren ser descentralizadas o con poder para la toma de decisiones, incluida la inversión, para estar a cargo de la intervención social.

Las condiciones necesarias para la aplicación del enfoque diferencial de derechos hacen pensar que se requiere de una reingeniería social e institucional que haga posible el piso adecuado para implementarlo, pues, si no, se corre el riesgo de acciones aisladas con inversiones poco proporcionadas a las realidades que se quieren modificar, con fuerte desgaste para los sujetos sociales involucrados y con competencia entre grupos discriminados para hacer exigibles sus derechos. Asimismo, se corre el riesgo de generar un sobreuso de los sistemas de justicia para la exigibilidad de derechos, cuyos pronunciamientos pasan a ser declarativos por la incapacidad del Estado de dar respuestas oportunas y pertinentes (Abramovich, 2004, p. 35).

\section{Conclusiones}

El escenario del posconflicto colombiano es un momento propicio para reconocer las diversas formas de discriminación que han afectado en el marco del conflicto y fuera de él a los sujetos sociales, entre ellos a las mujeres y a las minorías de género no hegemónico. Esto porque la finalización del conflicto armado, a través de la firma del acuerdo de paz, se constituye en un nuevo pacto social, en el cual se reconoce, entre otros, que el conflicto reprodujo diversas formas de discriminación social, la afectación en las víctimas y el efecto que tuvo en ellas, según sus particularidades, así como el compromiso estatal para la implementación del acuerdo a través de planes y políticas con la participación del conjunto de la sociedad colombiana. 
El acuerdo final incluye alrededor de 120 medidas que reconocen el impacto diferencial que el conflicto tuvo en las mujeres y la población LGBTI (Plataforma Cinco Claves, 2017). Estas medidas, en conjunto con otras de reconocimiento a las diversidades y de poblaciones afectadas ${ }^{15}$ por diversas formas de discriminación, serán implementadas a través de leyes y políticas públicas a nivel nacional y territorial. En otras palabras, es un escenario para hacer posible el enfoque diferencial de derechos en las políticas públicas del posconflicto colombiano.

El texto del acuerdo señala:

El Acuerdo está compuesto de una serie de acuerdos, que sin embargo constituyen un todo indisoluble, porque están permeados por un mismo enfoque de derechos, para que las medidas aquí acordadas contribuyan a la materialización de los derechos constitucionales de los colombianos y colombianas. El Acuerdo Final reconoce, sin discriminación alguna, la primacía de los derechos inalienables de la persona como fundamento para la convivencia en el ámbito público y privado, y a la familia como núcleo fundamental de la sociedad y los derechos de sus integrantes. La implementación del Acuerdo deberá regirse por el reconocimiento de la igualdad y protección del pluralismo de la sociedad colombiana, sin ninguna discriminación. En la implementación se garantizarán las condiciones para que la igualdad sea real y efectiva y se adoptarán medidas afirmativas en favor de grupos discriminados o marginados, teniendo en cuenta el enfoque territorial, diferencial y de género. (Gobierno de Colombia y las Farc-EP, 2016, p. 6)

15 El nuevo acuerdo para la paz señala en su página 3: "Subrayando que el nuevo Acuerdo Final presta especial atención a los derechos fundamentales de las mujeres, de los grupos sociales vulnerables como son los pueblos indígenas, las niñas, niños y adolescentes, las comunidades afrodescendientes y otros grupos étnicamente diferenciados; de los derechos fundamentales de los campesinos y campesinas y de los derechos esenciales de las personas en condición de discapacidad y de los desplazados por razones del conflicto; de los derechos fundamentales de las personas adultas mayores y de la población LGBTI" (Oficina del Alto Comisionado para la Paz, 2016). 
Así, numerosos desafíos se vislumbran para garantizar el éxito del proceso, y en particular, en materia de garantía de derechos y superación de las múltiples discriminaciones. Estos desafíos podrán ser superados en la medida que el Estado colombiano genere un diálogo social con participación y representación de los diversos grupos sociales, y que genere de esta forma alianzas que superen la fragmentación y polarización política que existe actualmente. Se requiere a su vez un ejercicio permanente de la sociedad civil y de la justicia colombiana, para garantizar que en la implementación de las políticas públicas del posconflicto se apliquen los estándares del derecho internacional de los derechos humanos. También, es necesario no solo garantizar la difusión del proceso, sino fomentar la participación ciudadana y realizar acciones de control y rendición de cuentas en el marco de los principios de transparencia y anticorrupción.

Las acciones que se adelanten deberán en la práctica articular de manera integral los enfoques de género, territorial y diferencial, para garantizar el criterio de universalidad y focalización de las políticas públicas y brindar una atención integral que permita la superación de las múltiples discriminaciones que atraviesan a los sujetos sociales.

El acuerdo de paz contempla la creación de un nuevo aparato institucional como soporte para las medidas que busca implementar; no obstante, es necesario garantizar un diálogo fluido con las instancias estatales preexistentes al acuerdo de paz, para evitar, por un lado, choques institucionales, y por otro, para que la inversión social que adelante el conjunto del Estado colombiano se revierta no solo en la superación del impacto del conflicto, sino en el desarrollo del país, expresado en un Estado que garantiza la igualdad sustantiva al conjunto de su diversa y plural sociedad.

De no superarse estos desafíos no solo los procesos de exigibilidad de derechos aumentarán en número en el sistema de justicia, sino muy probablemente el acuerdo de paz será uno más de los intentos de construcción inacabados en su propósito de garantizar el enfoque diferencial de derechos, particularmente para las mujeres y las minorías con género no hegemónico. 


\section{Referencias}

Abramovich, V. (2004). Una aproximación al enfoque de derechos en las estrategias y políticas de desarrollo de América Latina. Recuperado de http:// www.dhl.hegoa.ehu.es/ficheros/0000/0057/enfoque_de_dchos_en_estrategias_y_politicas_desarrollo_Am_Lat.pdf

Abramovich, V. (2009). De las violaciones masivas a los patrones estructurales: nuevos enfoques y clásicas tensiones en el Sistema Interamericano de Derechos Humanos. Revista Internacional de Derechos Humanos, SUR, 6(11). Recuperado de http://www.corteidh.or.cr/tablas/r24902.pdf

Campillo-Vélez, B. E. (2013). La ideología de género en el derecho colombiano. Díkaion, 22(1) 13-54. Recuperado de http://dikaion.unisabana.edu. co/index.php/dikaion/article/view/2784/3259

Naciones Unidas (1979). Convención sobre la Eliminación de Todas las Formas de Discriminación contra la Mujer (CEDAw). Recuperado de www. un.org/womenwatch/daw/cedaw/text/sconvention.htm

Comité de la CEDAW (s. f.). Recomendación General 28 relativa al artículo 2 de la Convención sobre la Eliminación de Todas las Formas de Discriminación contra la Mujer. Recuperado de http://www.acnur.org/fileadmin/Documentos/BDL/2012/8338.pdf?file=fileadmin/Documentos/BDL/2012/8338

Comité de la CEDAW (2017). General recommendation n. 35 on gender-based violence against women, updating general recommendation n. ${ }^{\circ} 19$. Recuperado de http://tbinternet.ohchr.org/Treaties/CEDAW/Shared \%20 Documents/1_Global/CEDAW_C_GC_35_8267_E.pdf

Cruz, J. (8 de mayo de 2007). Guadalupe. Igualdad: valemos lo mismo, pero somos diferentes. Cimac. Recuperado de http://www.cimacnoticias.com. $\mathrm{mx} /$ node $/ 51210$

De la Cruz, C. (1999). Guía metodológica para integrar la perspectiva de género en proyectos y programas de desarrollo. Emakunde-Instituto Vasco de la Mujer, Vitoria-Gasteiz. Recuperado de http://www.inmujeres. gub.uy/innovaportal/file/18616/1/12de_la_cruz_carmen._med_a_ged.pdf

De Lama, A. A. (2013). Discriminación múltiple. ADC (tomo LXVI, fasc. I). Recuperado de https://www.boe.es/publicaciones/.../abrir_pdf.php?... Discriminación_múltiple

Fernández, M. (2017). Aproximación a las temáticas de género en la jurisprudencia interamericana. Revista Argentina de Teoría Jurídica, 17. Recuperado de http://www.utdt.edu/ver_contenido.php?id_contenido= 12835\&id_item_menu=5858 
Fraser, N. (1997). Iustitia interrupta : reflexiones criticas desde la posición postsocialista. Siglo del Hombre Editores.

Fries, L. y Lacrampette, N., Feminismos, género y derecho, en Lacrampette, N. (ed.). (2013). Derechos Humanos y Mujeres: Teoría y Práctica. Centro de Derechos Humanos, Facultad de Derecho, Universidad de Chile. Recuperado de http://www.cdh.uchile.cl/media/publicaciones/pdf/101.pdf

Garay, N. (2008). Alcances sobre la discriminación racial o étnica y su vinculación histórica con la discriminación por sexo. Recuperado de https:// dialnet.unirioja.es/servlet/articulo? codigo $=3002234$

Gobierno de Colombia y Farc-EP (2016). Acuerdo Final para la Terminación del Conflicto y la Construcción de una Paz Estable y Duradera. Recuperado de http://www.altocomisionadoparalapaz.gov.co/procesos-y-conversaciones/Documentos\%20compartidos/24-11-2016NuevoAcuerdoFinal.pdf

Lemaitre, J. (2016). ¿Qué es una ideología de género? La silla vacía. Recuperado de http://lasillavacia.com/blogs/que-es-una-ideologia-de-genero-57494

Tarrés, M. L. (2013). A propósito de la categoría género: leer a Joan Scott. Estudios Sociológicos, XXXI(91), 380-390. Recuperado de https://www. revistas.ufg.br/fchf/article/viewFile/22406/13417

Osborne, R. y Molina, C. (2008). La evolución del concepto de género. Empiria: Revista de metodología de ciencias sociales, (15), 147-182. Recuperado de https://dialnet.unirioja.es/servlet/articulo?codigo=2686277

Plataforma Cinco Claves para un Tratamiento Diferencial de la Violencia Sexual en los Acuerdos sobre la Justicia Transicional en el Proceso de Paz. Equidad de género y derechos de las mujeres en el Acuerdo Final de Paz (2017). Recuperado de http://humanas.org.co/alfa/dat_particular/ar/ar_7354_q_Equidad-Genero-Mujeres-Acuerdo-final-1-1.pdf

Scott, J. W. (1990). El género: una categoría útil para el análisis histórico. En J. Amelang y M. Nash (eds.). Historia y género: Las mujeres en la Europa moderna y contemporánea. Recuperado de https://www.fundacionhenrydunant.org/images/stories/biblioteca/derechos_economicos_sociales_ culturales_genero/El\%20Genero \%20Una \%20Categoria \%20Util\%20 para $\% 20$ el $\% 20$ Analisis $\% 20$ Historico.pdf 
